Pacific

Journal of

Mathematics

ON UNIVALENT HARMONIC MAPPINGS AND MINIMAL SURFACES

Allen Weitsman

Volume 192 No. 1

January 2000 


\title{
ON UNIVALENT HARMONIC MAPPINGS AND MINIMAL SURFACES
}

\author{
Allen Weitsman
}

\begin{abstract}
If $S$ is the graph of a minimal surface, then when given parametrically by the Weierstrass representation, the first two coordinate functions give a univalent harmonic mapping. In this paper, the starting point is a univalent harmonic mapping $f$ of the unit disk $U$. A height function is defined on an appropriate Riemann surface over the range of $f$ which satisfies the minimal surface equation away from the branch points. This height function is then used to obtain function theoretic information about $f$.
\end{abstract}

\section{Introduction.}

Let $f$ be a univalent harmonic mapping of the unit disk $U$. By this it is meant not only that $f$ is $1-1$ and harmonic, but also that $f$ is sense preserving.

Harmonic univalent mappings were first studied in connection with minimal surfaces by E. Heinz $[\mathbf{H}]$. However, considerable interest in their function theoretic properties, quite apart from this connection, was generated by Clunie and Sheil-Small [CS-S].

Now, the Jacobian of $f(\zeta)$ is $J=\left|f_{\zeta}\right|^{2}-\left|f_{\bar{\zeta}}\right|^{2}$, and $f$ can be written

$$
f=h+\bar{g}
$$

where $h$ and $g$ are analytic in $U$. If $a(\zeta)$ is defined by

$$
a(\zeta)=\overline{f_{\bar{\zeta}}(\zeta)} / f_{\zeta}(\zeta)=g^{\prime}(\zeta) / h^{\prime}(\zeta),
$$

then $a(\zeta)$ is analytic and $|a(\zeta)|<1$ in $U$. We shall refer to $a(\zeta)$ as the analytic dilatation as opposed to the usual dilatation $f_{\bar{\zeta}} / f_{\zeta}$ in the theory of quasiconformal mappings.

The case where $a(\zeta)$ is a finite Blaschke product is of special interest since this case arises in taking Fourier series of step functions [S-S]. Their function theoretic properties have been studied in [HS2] as well as in $[\mathbf{S}-\mathbf{S}]$, and infinite Blaschke products have been considered in $[\mathbf{L}]$.

In the present paper we shall study a connection between harmonic mappings and the theory of minimal surfaces, and in $\S 4$ we use this to prove a special case of uniqueness for the Riemann mapping theorem of Hengartner 
and Schober [HS1]. As we have shown elsewhere, uniqueness fails in general $[\mathbf{W}]$.

\section{Definition of the height function and conjugate height function.}

Using the Weierstrass representation [O, p. 63] we shall associate with $f$, a minimal surface given parametrically in a simply connected subdomain $N \subseteq U$ where $a(\zeta)$ does not have a zero of odd order.

With $g$ and $h$ as in (1.1) we define up to an additive constant, a branch of

$$
F(\zeta)=2 i \int \sqrt{h^{\prime}(\zeta) g^{\prime}(\zeta)} d \zeta=2 i \int h^{\prime}(\zeta) \sqrt{a(\zeta)} d \zeta=2 i \int f_{\zeta}(\zeta) \sqrt{a(\zeta)} d \zeta
$$

Then, by (1.2) it follows that a branch of $F$ can be defined in $N$, and for $\zeta \in N$,

$$
\zeta \rightarrow(f(\zeta), \operatorname{Re} F(\zeta))
$$

gives a parametric representation of a minimal surface. Here we have identified $\mathbb{R}^{2}$ with $\mathbb{C}$ by $(x, y) \leftrightarrow(\operatorname{Re} f, \operatorname{Im} f)$.

Let $\hat{U}$ be the Riemann surface of the function $\sqrt{a(\zeta)}$. Then $\hat{U}$ has algebraic branch points corresponding to those points $\zeta \in U$ for which $a(\zeta)$ has a zero of odd order. Specifically, $\hat{U}$ can be concretely described (the analytic configuration [Sp, 69-74]) in terms of function elements $\left(\alpha, F_{\alpha}\right)$ where $\alpha \in U$, and $F_{\alpha}$ is a power series expansion of a branch of $F$ in a neighborhood of $\alpha$ if $a(\zeta)$ does not have a zero of odd order at $\zeta=\alpha$, and $F_{\alpha}$ a power series in $\sqrt{\zeta-\alpha}$ otherwise. The mapping $p:\left(\alpha, F_{\alpha}\right) \rightarrow \alpha$ is the projection of the surface so realized. The mapping $F$ may now be lifted to a mapping $\hat{F}$ on $\hat{U}$.

By continuation, we may induce a mapping $\hat{U} \rightarrow \tilde{U}$ to a surface $\tilde{U}$ with a real analytic structure defined in terms of elements $\left(\beta, \tilde{F}_{\beta}\right)$ with $\beta \in f(U)$ by $\alpha=f^{-1}(\beta)$ and $\tilde{F}_{\beta}=F_{\alpha} \circ f^{-1}$. We again define a projection by $\pi:\left(\beta, \tilde{F}_{\beta}\right) \rightarrow$ $\beta$.

We shall refer to a point $\hat{\zeta} \in \hat{U}$ to be over $\zeta$, if $p(\hat{\zeta})=\zeta$, and $\tilde{z} \in \tilde{U}$ to be over $z$ if $\pi(\tilde{z})=z$.

The harmonic mapping $f: U \rightarrow f(U)$ lifts to a mapping $\hat{f}: \hat{U} \rightarrow \tilde{U}$ which is $1-1$, onto, and satisfies the condition $\pi(\hat{f}(\hat{\zeta}))=f(p(\hat{\zeta}))$ for all $\zeta \in \hat{U}$. With these notations, we shall extend the meaning of (2.2). Thus

$$
\hat{\zeta} \rightarrow(\hat{f}(\hat{\zeta}), \operatorname{Re} \hat{F}(\hat{\zeta}))
$$

gives a parametric representation of a minimal surface in the sense that in a neighborhood of $\hat{\zeta} \in \hat{U} \backslash \mathcal{B}$ where $\mathcal{B}$ is the branch set, that is, the points 
above the zeros of $a$ of odd order, then (2.2) is the same as (2.3) computed in terms of local coordinates given by projection.

We may also define the surface nonparametrically on $\tilde{U} \backslash \tilde{\mathcal{B}}$, where $\tilde{\mathcal{B}}=$ $\hat{f}(\mathcal{B})$, as follows. Let $D$ be an open disk in $f(U)$ such that $f^{-1}(D)$ contains no zeros of $a$ of odd multiplicity. Let $w=\varphi(x, y)$ be the nonparametric description of the minimal surface corresponding to (2.2), that is, for $\zeta \in$ $f^{-1}(0)$ (cf. [HS3, p. 87]),

$$
\begin{aligned}
& x=\operatorname{Re} f(\zeta) \quad y=\operatorname{Im} f(\zeta), \\
& \varphi(x, y)=\operatorname{Re} F(\zeta) .
\end{aligned}
$$

Then, by continuation $\varphi$ lifts to a function $\tilde{\varphi}$ on $\tilde{U}$ which satisfies the minimal surface equation when computed in local coordinates given by projection off the branch set $\tilde{\mathcal{B}}$. We shall call $\tilde{\varphi}(\tilde{z})$ a height function corresponding to $f$. Finally, we define a conjugate height function $\tilde{\psi}(z)$ by solving locally

$$
\psi_{y}=\varphi_{x} / W, \psi_{x}=-\varphi_{y} / W \quad\left(W=\sqrt{1+\varphi_{x}^{2}+\varphi_{y}^{2}}\right)
$$

(cf. [F1, p. 344]) and lifting to $\tilde{U} \backslash \tilde{\mathcal{B}}$ as was done for $\varphi$. Let $\tilde{F}=\tilde{\varphi}+i \tilde{\psi}$. Then $\tilde{F}$ is real analytic and locally quasiconformal on $\tilde{U} \backslash \tilde{\mathcal{B}}$, with dilatation whose magnitude is $(W-1) /(W+1)$. The fact that $\tilde{\psi}$ and $\tilde{F}$ are well defined on $\tilde{U} \backslash \tilde{B}$ follows from Theorem 1 .

A glossary of terminology is given schematically in Figure 1.

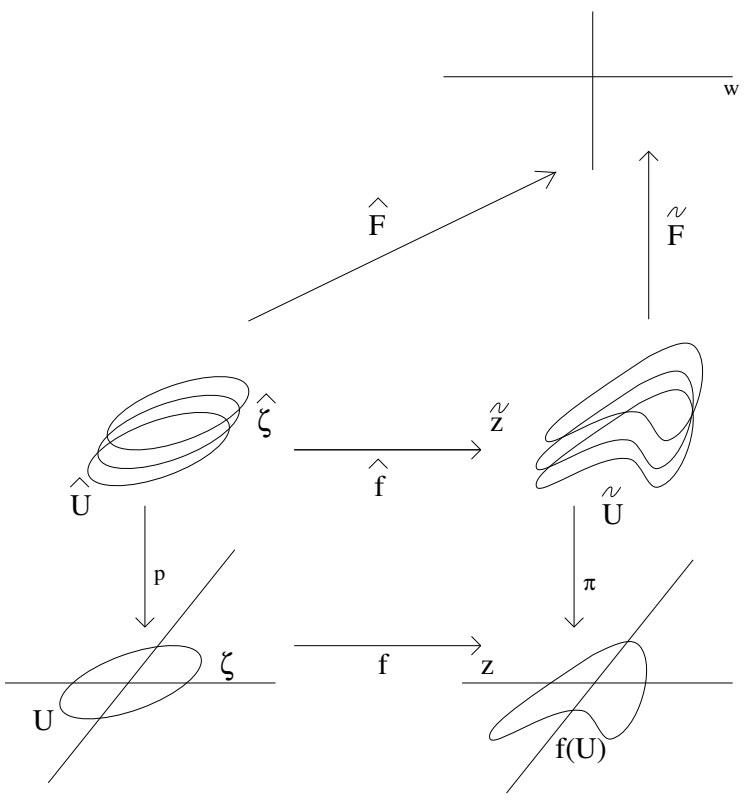

Figure 1. 
Theorem 1. With the above notations, $\hat{F}=\tilde{F} \circ \hat{f}+C$ for some constant $C$.

Proof. Let $D$ be an open disk in $f(U)$ such that $f^{-1}(D)$ contain no zeros of odd multiplicities of $a$. We fix a branch of $\sqrt{a}$ in $f^{-1}(D)$, and consider $\hat{\varphi}(\hat{\zeta})+i \hat{\psi}(\hat{\zeta})=\hat{F}(\hat{\zeta})$ for points in a component of $\hat{U}$ over $f^{-1}(D)$, and $\tilde{\varphi}(\tilde{z})+i \tilde{\psi}(\tilde{z})=\tilde{F}(\tilde{z})$ for points in a component of $\tilde{U}$ over $D$. Since we shall compute in local coordinates given by projection, to reduce notation in this proof, we shall subsequently write $\hat{F}, \hat{\varphi}, \hat{\psi}$ in place of $\hat{F} \circ p^{-1}, \hat{\varphi} \circ p^{-1}, \hat{\psi} \circ p^{-1}$, and $\tilde{F}, \tilde{\varphi}, \tilde{\psi}$ in place of $\tilde{F} \circ \pi^{-1}, \tilde{\varphi} \circ \pi^{-1}, \tilde{\psi} \circ \pi^{-1}$ respectively. With this notation, by (2.4) we have that

$$
\hat{\varphi}=\tilde{\varphi} \circ f
$$

so it suffices to show that

$$
\hat{\psi}=\tilde{\psi} \circ f+C .
$$

The result then follows from continuation.

In fact, since $\hat{\varphi}+i \hat{\psi}$ is analytic in $f^{-1}(D)$, it follows from (2.6) that to prove (2.7) it suffices to show that $\tilde{F} \circ f$ is analytic in $f^{-1}(D)$.

We first record the relationship between $a(\zeta)$ of $(1.2)$ and $W(z)(z=f(\zeta))$ of (2.5). This is given by [O, p. 105], [HS3, pp. 87-88] as

$$
|a|=\frac{W-1}{W+1} \text {. }
$$

Now,

$$
(\tilde{F} \circ f)_{\bar{\zeta}}=\tilde{F}_{z} f_{\bar{\zeta}}+\tilde{F}_{\bar{z}} \bar{f}_{\bar{\zeta}}=\tilde{F}_{z} f_{\bar{\zeta}}+\tilde{F}_{\bar{z}} \overline{\left(f_{\zeta}\right)} .
$$

A simple computation using (2.5) gives

$$
F_{z}=\frac{W+1}{W} \varphi_{z}, \quad F_{\bar{z}}=\frac{W-1}{W} \varphi_{\bar{z}} .
$$

When used in (2.9) these give

$$
(\tilde{F} \circ f)_{\bar{\zeta}}=\frac{W+1}{W} \tilde{\varphi}_{z} f_{\bar{\zeta}}+\frac{W-1}{W} \tilde{\varphi}_{\bar{z}} \overline{\left(f_{\zeta}\right)} .
$$

Again, a direct computation gives

$$
\tilde{\varphi}_{z}=\frac{\hat{\varphi}_{\zeta} \overline{\left(f_{\zeta}\right)}-\hat{\varphi}_{\bar{\zeta}} \overline{\left(f_{\bar{\zeta}}\right)}}{\left|f_{\zeta}\right|^{2}-\left|f_{\bar{\zeta}}\right|^{2}}, \quad \tilde{\varphi}_{\bar{z}}=\frac{\hat{\varphi}_{\bar{\zeta}} f_{\zeta}-\hat{\varphi}_{\zeta} f_{\bar{\zeta}}}{\left|f_{\zeta}\right|^{2}-\left|f_{\bar{\zeta}}\right|^{2}}
$$

When used in (2.10) this gives

$$
(\tilde{F} \circ f)_{\bar{\zeta}}=\frac{1}{W\left(\left|f_{\zeta}\right|^{2}-\left|f_{\bar{\zeta}}\right|^{2}\right)}\left(2 \hat{\varphi}_{\zeta} f_{\bar{\zeta}} \overline{\left(f_{\zeta}\right)}+\hat{\varphi}_{\bar{\zeta}}\left|f_{\zeta}\right|^{2}\left(W-1-\frac{\left|f_{\bar{\zeta}}\right|^{2}}{\left|f_{\zeta}\right|^{2}}(W+1)\right)\right) .
$$


Now, by (1.2), (2.1), and (2.8) we have,

$$
\hat{\varphi}_{\zeta}=i g^{\prime} / \sqrt{a}, \hat{\varphi}_{\bar{\zeta}}=-i \overline{g^{\prime}} / \overline{\sqrt{a}}, f_{\zeta}=g^{\prime} / a, f_{\bar{\zeta}}=\overline{g^{\prime}},
$$

and

$$
W-1-\frac{\left|f_{\bar{\zeta}}\right|^{2}}{\left|f_{\zeta}\right|^{2}}(W+1)=W-1-|a|^{2}(W+1)=2(W-1) /(W+1) .
$$

Substituting into (2.11) we obtain

$$
\begin{aligned}
(\tilde{F} \circ f)_{\bar{\zeta}} & =\frac{1}{W\left(\left|f_{\zeta}\right|^{2}-\left|f_{\bar{\zeta}}\right|^{2}\right)}\left(\frac{2 i g^{\prime}\left(\overline{g^{\prime}}\right)^{2}}{\sqrt{a} \bar{a}}-\frac{2 i \overline{g^{\prime}}\left|g^{\prime}\right|^{2}}{\overline{\sqrt{a}}|a|^{2}}\left(\frac{W-1}{W+1}\right)\right) \\
& =0 .
\end{aligned}
$$

Thus, $\tilde{F} \circ f$ is analytic and (2.7) follows.

\section{The height function corresponding to Poisson integrals of step functions.}

Let $\mathcal{P}$ be a polygon with vertices $c_{1}, \ldots, c_{n}$ given cyclically, and in order induced by a positive orientation of $\partial \mathcal{P}$. Let $f$ be the Poisson integral of a step function on $\partial U$ having values $c_{1}, \ldots, c_{n}$ and suppose that $f$ is then a univalent harmonic mapping, $f: U \rightarrow \mathcal{P}$. If $\mathcal{P}$ is convex, for example, this will always be the case $[\mathbf{C}],[\mathbf{K}]$. The analytic dilatation $a(\zeta)$ for such mappings were studied in $[\mathbf{H S 2}]$ and $[\mathbf{S}-\mathbf{S}]$. In general, $a(\zeta)$ is a Blaschke product of order at most $n-2$, and of order precisely $n-2$ if $\mathcal{P}$ is convex [S-S, pp. 469, 473].

We shall now explore the boundary behavior of height functions corresponding to such mappings. The prototype for this is Scherk's minimal surface over the square $-\pi / 2<x<\pi / 2,-\pi / 2<y<\pi / 2$, given by

$$
\psi(x, y)=\log (\cos x / \cos y)
$$

which tends to $+\infty$ and $-\infty$ over alternate sides. It seems remarkable that this type of behavior persists in general for height functions corresponding to all such $f$ described above.

Theorem 2. Let $\mathcal{P}$ be a polygon having vertices $c_{1}, \ldots, c_{n}$ given cyclically, and ordered by a positive orientation on $\partial \mathcal{P}$. Let $f$ be a univalent harmonic mapping of $U$ such that $f$ is the Poisson integral of a step function having the ordered sequence $c_{1}, \ldots, c_{n}$ as its values. Then the analytic dilatation $a(\zeta)$ of $f$ is a finite Blaschke product of order at most $n-2, f(U)=\mathcal{P}$, and if $\varphi$ is a height function for $f$, then $\varphi$ tends to $+\infty$ or $-\infty$ at points over the open segments making up the sides of $\mathcal{P}$. If $\mathcal{P}$ is convex, then $+\infty$ and $-\infty$ alternate on adjacent sides. 
Proof. That $a(\zeta)$ is a Blaschke product of order at most $n-2$ and $f(U)=$ $\mathcal{P}$ follow from general properties of Poisson integrals [S-S, p. 469], [HS2, p. 203].

Let $f=h+\bar{g}$ as in (1.1). Then we may write $h^{\prime}$ and $g^{\prime}$ in the form [S-S, pp. 460-461]

$$
h^{\prime}(\zeta)=\sum_{k=1}^{n} \frac{\alpha_{k}}{\zeta-\zeta_{k}}, g^{\prime}(\zeta)=-\sum_{k=1}^{n} \frac{\overline{\alpha_{k}}}{\zeta-\zeta_{k}},
$$

where $\alpha_{k} \neq 0, \quad k=1, \ldots, n$.

With $F$ as in (2.1), we are then interested in the branches of

$$
F(\zeta)=-2 \int \sqrt{\sum_{k=1}^{n} \frac{\alpha_{k}}{\zeta-\zeta_{k}} \sum_{k=1}^{n} \frac{-\overline{\alpha_{k}}}{\zeta-\zeta_{k}}} d \zeta
$$

as $\zeta \rightarrow \zeta_{k}, \quad k=1, \ldots, n$. The cluster sets for the nontangential approaches to points over the $\zeta_{k}$ give the points lying over the open segments making up the sides of $\mathcal{P}$.

Thus, take a vertex $\zeta_{j}$, and an open segment $l_{j}$ of $\partial \mathcal{P}$ corresponding to it. Then, as $\zeta \rightarrow \zeta_{j}$,

$$
\sum_{k=1}^{n} \frac{\alpha_{k}}{\zeta-\zeta_{k}} \sum \frac{-\overline{\alpha_{k}}}{\zeta-\zeta_{k}}=\frac{\left|\alpha_{j}\right|^{2}}{\left(\zeta-\zeta_{j}\right)^{2}}(1+o(1)),
$$

and hence, by (3.2), a branch of $F$ satisfies

$$
F(\zeta)= \pm 2\left|\alpha_{j}\right| \log \left(\zeta-\zeta_{j}\right)+o(1)
$$

as $\zeta \rightarrow \zeta_{j}$, for a fixed branch of the log. Suppose the fixed branch of (3.3) has minus sign, and let $\phi(z)=\operatorname{Re} F \circ f^{-1}(z)$ be a corresponding branch in $\mathcal{P}$ for points near the corresponding side $l_{j}$. Now suppose $\mathcal{P}$ is convex and $F(\zeta)$ is analytically continued to an adjacent point, say $\zeta_{j+1}$, so that $\phi$ is then continued to a corresponding side $l_{j+1}$ having common endpoint $c_{j}$ with $l_{j}$. Since $\phi \rightarrow-\infty$ as $z \rightarrow l_{j}$, it remains to show that $\phi \rightarrow+\infty$ as $z \rightarrow l_{j+1}$. This effect has been noted for minimal surfaces [JS], and can be accomplished by a simple barrier argument. I thank Professor Finn for pointing this out.

Let $0<\beta<\pi$ be the angle in $\mathcal{P}$ between $l_{j}$ and $l_{j+1}$. Suppose that $\phi \rightarrow-\infty$ on both open segments $l_{j}$ and $l_{j+1}$. Since $\phi$ satisfies the minimal surface equation, $\phi$ can only tend to $-\infty$ over line segments $[\mathbf{O}$, p. 102] Since we make no assumption at the common endpoint $c_{j}$, in order to get a contradiction we must show that $\phi \rightarrow-\infty$ at $c_{j}$ as well. We may assume that $c_{j}=(\pi / 2,0)$, and $l_{j}, l_{j+1}$ make the angle $\beta$ symmetrically with respect to the $x$ axis, opening toward the origin. Let $0<\varepsilon<(\pi / 2) \cot (\beta / 2)$ be small enough so that the isosceles triangle $N$ formed by the sector and the line $x=\pi / 2-\varepsilon$ has the given branch of $F$ single valued. Then, two of 
the sides of $N$ are contained in the segments $l_{j}$ and $l_{j+1}$, and the third is $x=\pi / 2-\varepsilon,-\delta<y<\delta$, where $\delta=\varepsilon \tan (\beta / 2)$. If $\psi$ is the height function for Scherk's surface given by (3.1), then for any $M>0$, clearly

$$
\phi(x, y)<-\psi(x-\pi+\varepsilon, y)-M
$$

on $\partial N \backslash\left\{c_{j}\right\}$. By the extended maximum principle [F1, pp. 342-343], it follows that (3.4) holds thoughout $N$. Since $M>0$ was arbitrary, it follows that $\phi \equiv-\infty$ on $N$, a contradiction. Thus $\phi=+\infty$ on $l_{j+1}$.

\section{An application to the Riemann mapping theorem.}

One of the most basic results in the theory of univalent harmonic mappings is the Riemann mapping theorem of Hengartner and Schober [HS1].

Theorem A. Let $D$ be a bounded simply connected domain whose boundary is locally connected. Fix $w_{0} \in D$, and let $a(\zeta)$ be analytic in $U$, with $a(U) \subseteq U$. Then there exists a univalent harmonic mapping $f$ with the following properties.

a) $f$ maps $U$ into $D$ and $f(0)=w_{0}, f_{z}(0)>0$.

b) $f$ satisfies the equation $\overline{\left(f_{\bar{\zeta}}\right)}=a f_{\zeta}$.

c) Except for a countable set $E \subseteq \partial U$, the unrestricted limit $f^{*}\left(e^{i t}\right)=$ $\lim _{\zeta \rightarrow e^{i t}} f(\zeta)$ exists and belongs to $\partial D$.

d) The one sided limits $\lim _{\tau \rightarrow t^{+}} f *\left(e^{i \tau}\right), \lim _{\tau \rightarrow t^{-}} f^{*}\left(e^{i \tau}\right)$ through values of $e^{i \tau} \notin$ $E$ exist and belong to $\partial D$; for $e^{i t} \notin E$ they are equal and for $e^{i t} \in E$ they are different.

e) The cluster set of $f$ at $e^{i t} \in E$ is the straight line segment joining the left and right limits in $\mathrm{d}$ ).

If in Theorem $\mathrm{A}$, the set $D$ is convex, and $a(\zeta)$ is a finite Blaschke product, one can say more [HS2, p. 203], [S-S, p. 473].

Theorem B. Let $f$ be as in Theorem $A$ with $D$ bounded and convex, and $a(\zeta)$ a Blaschke product of order $n-2$. Then $f(U)$ is a polygon with $n$ vertices all of which lie on $\partial D$.

We shall prove uniqueness in the case $a(\zeta)=\zeta^{n}$ and $D$ convex. The case of uniqueness when $D=U$ and $a(\zeta)=\zeta$ was done in [HS2, p. 204].

The proof involves a combinatorial argument with the level sets of the height function. Such arguments are often useful in the theory of partial differential equation, and in particular the minimal surface equation [F1], [FO], [JS], [Se].

Theorem 3. The solution $f(\zeta)$ to the Riemann mapping theorem above with $D$ convex and

$$
a(\zeta)=\zeta^{n-2}
$$


is unique for each $n=3,4, \ldots$

Proof. Let $f_{1}$ and $f_{2}$ be Riemann mappings corresponding to $D$. We may assume $f_{1}(0)=f_{2}(0)=0$. Let $\Delta$ be a disk centered at 0 , and contained in $f_{1}(U) \cap f_{2}(U)$.

If $n$ is even, then $\hat{U}=U$ and if $n$ is odd $\hat{U}$ is a two sheeted cover of $U$ with branch point over 0 . Similarly, if $\tilde{U}_{1}$ corresponds to $f_{1}(U)$ and $\tilde{U}_{2}$ to $f\left(U_{2}\right)$, then $\tilde{U}_{1}$ and $\tilde{U}_{2}$ are one or two sheeted according as $n$ is even or odd. We consider the case where $n$ is odd. The even case goes the same way, but is simpler since one can bypass discussion of Riemann surfaces.

Let $\varphi_{j}, \psi_{j}, \tilde{\varphi}_{j}, \tilde{\psi}_{j}, \tilde{F}_{j}, \tilde{U}_{j}, \pi_{j}, \quad j=1,2$ be the quantities of $\S 2$ defined for $f_{1}$ and $f_{2}$ respectively. We may assume that $\tilde{F}_{1}(\tilde{0})=\tilde{F}_{2}(\tilde{0})=0$. If $\tilde{\Delta}$ represents the Riemann surface of $\sqrt{z}$ over $\Delta$, then we may consider $\tilde{\Delta} \subseteq \tilde{U}_{1}$ and $\tilde{\Delta} \subseteq \tilde{U}_{2}$, so that $\tilde{F}_{1}$ and $\tilde{F}_{2}$ may both be considered as defined for all $\tilde{z} \in \tilde{\Delta}$. For brevity of notation, we shall write $\tilde{F}$ for $\tilde{F} \circ \pi^{-1}$.

Since the analytic dilatation for $f_{1}(\zeta)$ and $f_{2}(\zeta)$ is 0 when $\zeta=0$, it follows from (1.2), (4.1), and a) of Theorem A, that

$$
f_{j}(\zeta)=c_{j} \zeta(1+o(1)) \quad\left(\zeta \rightarrow 0, c_{j}>0, j=1,2\right) .
$$

Then, from (2.1), (4.1), (4.2), and Theorem 1 we may take determinations of $\tilde{F}_{1}$ and $\tilde{F}_{2}$ in $\tilde{\Delta}$ so that

$$
\tilde{\varphi}_{j}(z)+i \tilde{\psi}_{j}(z)=\tilde{F}_{j}(z)=d_{j} z^{n / 2}(1+o(1)) \quad(j=1,2 \quad z \rightarrow 0)
$$

with $d_{1}, d_{2}>0$ and $z^{n / 2}$ is some fixed branch.

Having thus fixed branches in (4.3) we may then take a constant $\lambda>0$ such that

$$
\tilde{F}_{1}(z)-\lambda \tilde{F}_{2}(z / \lambda)=C z^{\frac{p+2}{2}}(1+o(1)) \quad(z \rightarrow 0)
$$

for some constant $C$ and integer $p \geq n$. We suppose $\lambda \geq 1$; otherwise we interchange $\tilde{F}_{1}$ and $\tilde{F}_{2}$. Now, the change from $F(z)$ to $\lambda F(z / \lambda)$ corresponds to replacing $f$ by $\lambda f$. Then the analytic dilatation is unchanged, and following the change in (2.1) it gives the parametrization $\zeta \rightarrow(\lambda f(\zeta), \operatorname{Re} \lambda F(\zeta))$.

Let $\varphi_{3}, \psi_{3}, \tilde{\varphi}_{3}, \tilde{\psi}_{3}$ correspond to $f_{3}=\lambda f_{2}$ so that $f_{3}(U)$, is nothing more than $f_{1}(U)$ dilated by the constant $\lambda \geq 1$, and (4.5) becomes

$$
\tilde{F}_{1}(\tilde{z})-\tilde{F}_{3}(\tilde{z})=C z^{\frac{p+2}{2}}(1+o(1)) \quad(z \rightarrow 0) .
$$

Case 1 . $C=0$ for every $p$. Since $\tilde{F}_{1}\left(z^{2}\right)-\tilde{F}_{3}\left(z^{2}\right)$ is real analytic, then $\tilde{F}_{1} \equiv$ $\tilde{F}_{3}$. Thus, in particular $\lambda=1$ and $f_{1}(U)=f_{3}(U)=\mathcal{P}$. In order to show that $f_{1} \equiv f_{3}$ we use the subordination principle of [BHH, p. 170]. Briefly, since $\mathcal{P}$ is a convex polygon by Theorem $\mathrm{B}$, and $\left(f_{1}\right)_{z}(0),\left(f_{3}\right)_{z}(0)>0$, we may apply the argument principle in $[\mathbf{B H H}$, p. 170] to

$$
G(z)=\left(f_{3}\right)_{z}(0) f_{1}(z)-\left(f_{1}\right)_{z}(0) f_{3}(z)
$$


to deduce that $\left(f_{1}\right)_{z}(0)=\left(f_{3}\right)_{z}(0)$. Then, another application of the argument principle as in $[\mathbf{B H H}]$ to $G_{\varepsilon}(z)=(1+\varepsilon) f_{1}(z)-f_{3}(z)(\varepsilon \rightarrow 0)$ shows that $f_{1} \equiv f_{3}$.

Case 2. $C \neq 0$ for some $p \geq n$. In this case, near the origin on $\tilde{\Delta}$, by (4.5) there are $2 p+4$ level curves $\tilde{\varphi}_{1}-\tilde{\varphi}_{3}=0$ emanating from $\tilde{0}$. Between the level curves, $\tilde{\varphi}_{1}-\tilde{\varphi}_{3}$ alternates in sign. In order to analyze the component sets between the level sets, we must modify $f_{3}$.

Let $\eta_{1}, \eta_{2}, \ldots$ be homeomorphisms of $|\zeta|=1$ onto the boundary of $\lambda D$, which converge to the (step function) boundary values of $f_{3}$, and let $f_{3}^{(n)}$, $n=1,2, \ldots$ their corresponding Poisson integrals so that $f_{3}^{(n)} \rightarrow f_{3}$ uniformly on compact subsets of $U$.

The level sets of $\tilde{\varphi}_{1}-\tilde{\varphi}_{3}=0$ create $2 p+4$ disjoint component open sets $O_{1}, O_{2}, \ldots, O_{2 p+4}$ where $\tilde{\varphi}_{1}-\tilde{\varphi}_{3}>0$ in $O_{2 j-1}$ and $\tilde{\varphi}_{1}-\tilde{\varphi}_{3}<0$ in $O_{2 j}$ for $j=1, \ldots, p+2$. These components alternate in position around the origin.

For $\varepsilon>0$ we can find nonempty components at $O_{1}(\varepsilon), O_{2}(\varepsilon), \ldots, O_{2 p+4}(\varepsilon)$ where $\tilde{\varphi}_{1}-\tilde{\varphi}_{3}^{(n)}>\varepsilon$ in $O_{2 j-1}(\varepsilon), \tilde{\varphi}-\tilde{\varphi}_{3}^{(n)}=\varepsilon$ on $\partial O_{2 j-1}(\varepsilon), \tilde{\Delta} \cap O_{2 j-1}(\varepsilon) \subseteq$ $O_{2 j-1}, j=1, \ldots, 2 p$, and analogous statements hold for $O_{2 j}(\varepsilon), j=1, \ldots$, $p+2$.

Now, $f_{3}^{(j)}(U)=\lambda D$, so by the maximum principle for solutions to the minimal surface equation, the level sets forming the boundaries of the $O_{j}(\varepsilon)$ 's must extend to points over the boundary of $\mathcal{P}=f_{1}(U)$. As in [FO, pp. 357358], we observe that since $\tilde{F}_{1}$ is $\pm \infty$ over the sides of $\mathcal{P}$ by Theorem 2 , if a component $O_{j}(\varepsilon)$ has a boundary point over an interior point of a side of $\mathcal{P}$, then the boundary must contain that side. Since, by Theorem B, $\mathcal{P}$ has $n$ sides, then $\tilde{\mathcal{P}}=\pi_{1}^{-1}(\mathcal{P})$ has $2 n$ sides. This implies that there are at most $2 n$ sets $O_{j}(\varepsilon)$ whose boundaries have interior points over $\partial \mathcal{P}$. If $O_{j}(\varepsilon)$ were a component whose boundary contained no points over $\partial \mathcal{P}$, then its boundary could only be interior points over $\mathcal{P}$, or vertices. As pointed out in [FO, p. 358], this is impossible by a theorem of Finn [F1, pp. 342-343]. Thus, $2 p+4 \leq 2 n$. Since $p \geq n$, we obtain a contradiction and the theorem is proved.

\section{References}

[BHH] D. Bshouty, N. Hengartner and W. Hengartner, A constructive method for starlike harmonic mappings, Numer. Math., 54 (1988), 167-178.

[C] G. Choquet, Sur un type de transformation analytique généralisant la représentation conforme et définie an moyen de fonctions harmoniques, Bull. Sci. Math., 69 (1945), 156-165.

[CS-S] J. Clunie and T. Sheil-Small, Harmonic univalent functions, Ann. Acad. Sci. Fenn. Ser. AI Math., 9 (1984), 3-25. 
[F1] R. Finn, New estimates for equations of minimal surface type, Arch. Rat. Mech. Anal., 14 (1963), 337-375.

[F2] Remarks relevant to minimal surfaces and to surfaces of prescribed mean curvature, J. d'Analyse Math., 14 (1965), 139-160.

[FO] R. Finn and R. Osserman, On the Gauss curvature of non-parametric minimal surfaces, J. d'Analyse Math., 12 (1964), 351-364.

$[\mathrm{H}] \quad$ E. Heinz, Über die Losungen der Minimalflachengleichung, Nachr. Akad. Wiss. Gottingen Math. Phys., K1 (1952), 51-56.

[HS1] W. Hengartner and G. Schober, Harmonic mappings with given dilatation, J. Lond. Math. Soc., 33(2) (1986), 473-483.

[HS2] _ On the boundary behavior of orientation-preserving harmonic mappings, Complex Variables, 5 (1986), 197-208.

[HS3] _ Curvature estimates for some minimal surfaces, in 'Complex Analysis', Birkhaüser Verlag, 1988, 87-100.

[JS] H. Jenkins and J. Serrin, Variational problems of minimal surface type II. Boundary value problems for the minimal surface equation, Arch. Rat. Mech. Anal., 21 (1965/66), 321-342.

[K] H. Kneser, Lösung der Aufgabe 41, Jahresber. Deutsch. Math. Verein., 35 (1925), 123-124.

[L] R. Laugesen, Planar harmonic maps with inner and Blaschke dilatations, J. Lond. Math. Soc., 56(2) (1997), 37-48.

[O] R. Osserman, A Survey of Minimal Surfaces, Dover, 1986.

[Se] J. Serrin, A priori estimates for solutions of the minimal surface equation, Arch. Rat. Mech. Anal., 14 (1963), 376-383.

[S-S] T. Sheil-Small, On the Fourier series of a step function, Mich. Math. J., 36 (1989), 459-475.

[Sp] G. Springer, Introduction to Riemann Surfaces, Addison-Wesley, 1957.

[W] A. Weitsman, A counterexample to uniqueness in the Riemann mapping theorem for univalent harmonic mappings, Bull. Lond. Math. Soc., 31 (1999), 87-89.

Received March 9, 1998.

Purdue University

West LAFAYETTE, IN 47907-1395

E-mail address: weits@math.purdue.edu 